\title{
Structure and Transport of Solvent Ligated Octahedral Mg-ion in an Aqueous Battery Electrolyte
}

\section{Thejus R. Kartha and Bhabani S. Mallik*}

Department of Chemistry, Indian Institute of Technology Hyderabad, Kandi-502285, Sangareddy, Telangana, India

*bhabani@chy.iith.ac.in
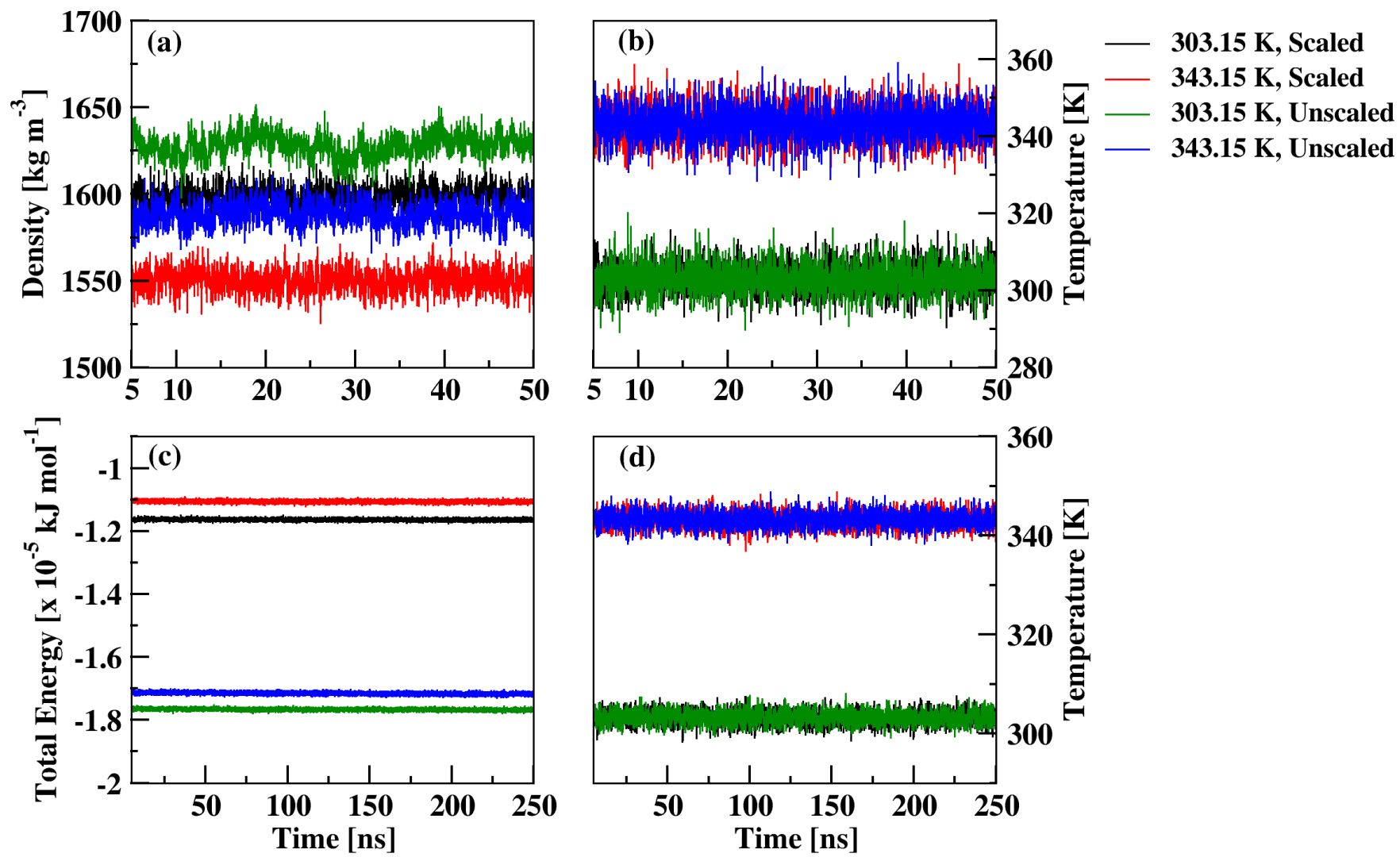

Figure S1. The convergence of physical properties after molecular dynamics simulations at $300 \mathrm{~K}$ : (a) Density through $50 \mathrm{~ns}$ of NPT (b) Temperature through $50 \mathrm{~ns}$ of NVT (c) Total energy through $250 \mathrm{~ns}$ of NVE (d) Temperature through $250 \mathrm{~ns}$ of NVE. 

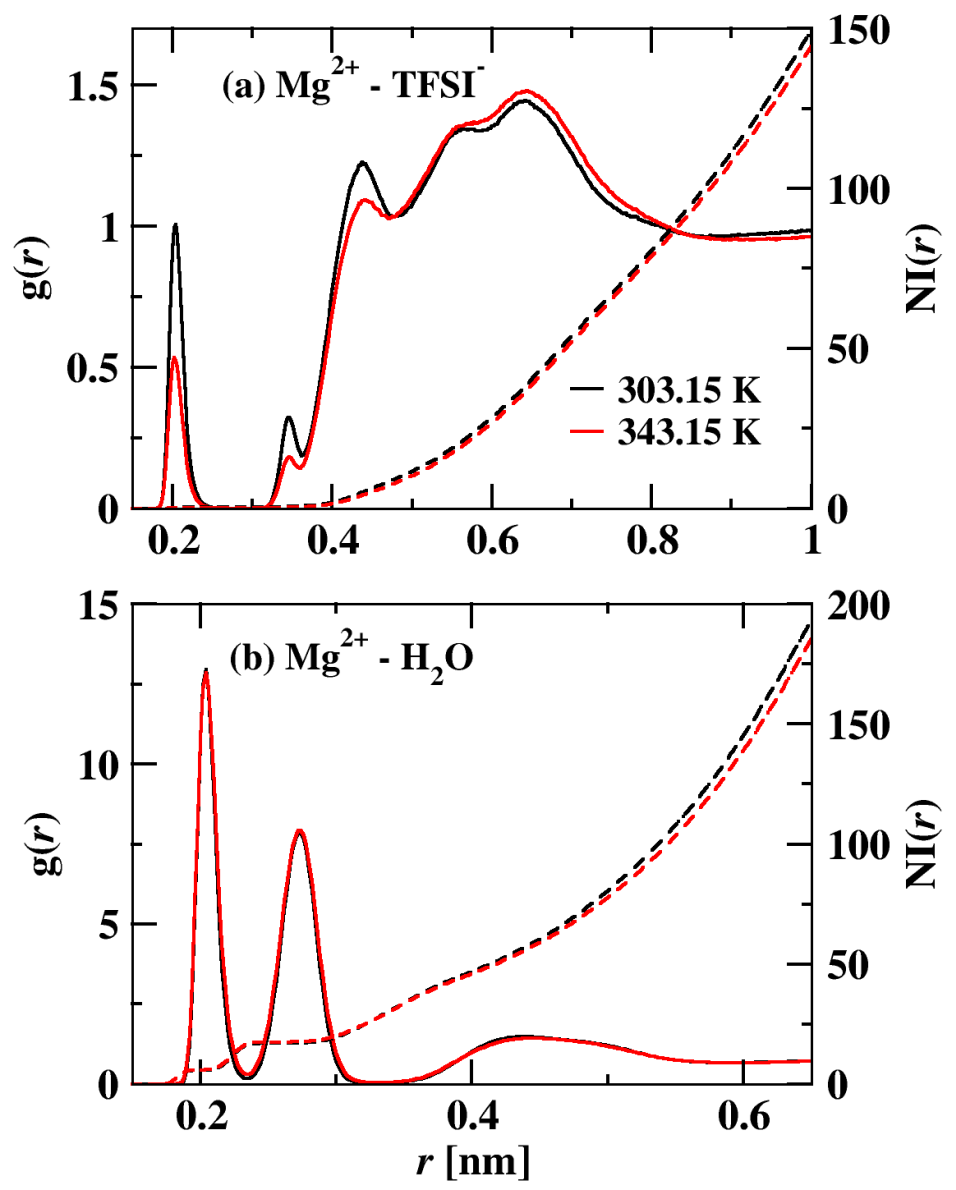

Figure S2. $\mathrm{RDF}$ of (a) $\mathrm{Mg}^{2+}$ - centre of mass of TFSI' and (b) $\mathrm{Mg}^{2+}$ - centre of mass of $\mathrm{H}_{2} \mathrm{O}$.

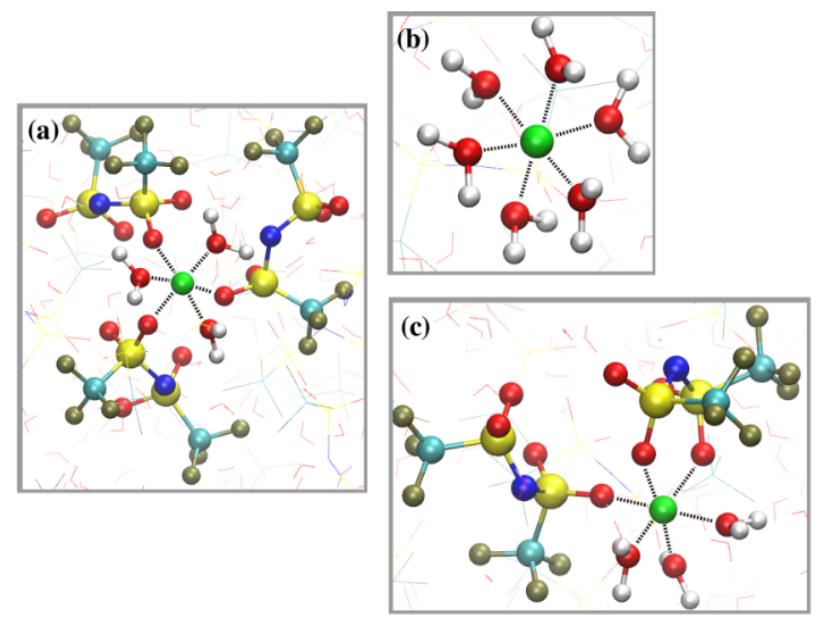

Figure S3. Snapshots of the solvation environment of the $\mathrm{Mg}^{2+}$ in the unscaled cases. (a) $\left[\mathrm{Mg}\left(\mathrm{H}_{2} \mathrm{O}\right)_{3}(\operatorname{trans}-\mathrm{TFSI})_{3}\right]$ (b) $\left[\mathrm{Mg}\left(\mathrm{H}_{2} \mathrm{O}\right)_{6}\right](\mathrm{c})\left[\mathrm{Mg}\left(\mathrm{H}_{2} \mathrm{O}\right)_{3}(\right.$ cis-TFSI)(trans-TFSI) $]$. 


\section{Non-Bonded Force field parameters}

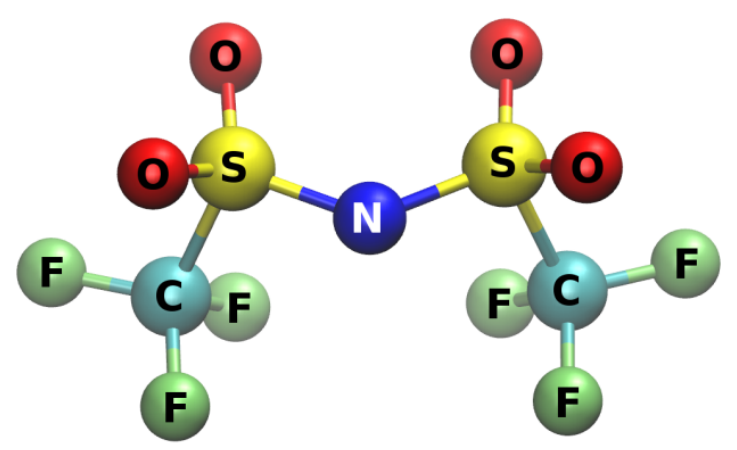

\begin{tabular}{llll}
\hline Atom & $\boldsymbol{\sigma}[\mathbf{n m}]$ & $\boldsymbol{\varepsilon}\left[\mathbf{k J ~ m o l}^{-\mathbf{1}}\right]$ & Charge (unscaled) \\
\hline $\mathbf{M g}$ & 0.141225 & 3.74342 & 2.000000 \\
$\mathbf{N}$ & 0.325000 & 0.711280 & -0.846520 \\
$\mathbf{S}$ & 0.356359 & 1.046000 & +1.327211 \\
$\mathbf{O}$ & 0.295992 & 0.878640 & -0.623515 \\
$\mathbf{C}$ & 0.339967 & 0.457730 & +0.204906 \\
$\mathbf{F}$ & 0.311815 & 0.255224 & -0.120609 \\
\hline
\end{tabular}




\section{Bonded Force Field Parameters (GROMACS .itp file, charges are not scaled)}

\section{TFSI $^{-}$}

\begin{tabular}{|c|c|c|c|c|c|c|c|}
\hline \multicolumn{8}{|c|}{ [ atoms ] } \\
\hline $\mathrm{nr}$ & type & resi & res & atom & $\operatorname{cgn} r$ & charge & mass \\
\hline 1 & $\mathrm{f}$ & 1 & TFS & F1 & 1 & -0.120609 & 19.00000 \\
\hline 2 & c3 3 & 1 & TFS & $\mathrm{C} 1$ & 2 & 0.204906 & 12.01000 \\
\hline 3 & $\mathrm{f}$ & 1 & TFS & F2 & 3 & -0.120609 & 19.00000 \\
\hline 4 & $\mathrm{f}$ & 1 & TFS & F3 & 4 & -0.120609 & 19.00000 \\
\hline 5 & sy & 1 & TFS & S1 & 5 & 1.327211 & 32.06000 \\
\hline 6 & 0 & 1 & TFS & 03 & 6 & -0.623515 & 16.00000 \\
\hline 7 & 0 & 1 & TFS & 04 & 7 & -0.623515 & 16.00000 \\
\hline 8 & ne & 1 & TFS & N1 & 8 & -0.846520 & 14.01000 \\
\hline 9 & s6 & 1 & TFS & s2 & 9 & 1.327211 & 32.06000 \\
\hline 10 & $\circ$ & 1 & TFS & 01 & 10 & -0.623515 & 16.00000 \\
\hline 11 & 0 & 1 & TFS & $\mathrm{O} 2$ & 11 & -0.623515 & 16.00000 \\
\hline 12 & c3 & 1 & TFS & $\mathrm{C} 2$ & 12 & 0.204906 & 12.01000 \\
\hline 13 & $f$ & 1 & TFS & F5 & 13 & -0.120609 & 19.00000 \\
\hline 14 & $\mathrm{f}$ & 1 & TFS & F6 & 14 & -0.120609 & 19.00000 \\
\hline 15 & $\mathrm{f}$ & 1 & TFS & F4 & 15 & -0.120609 & 19.00000 \\
\hline
\end{tabular}

\begin{tabular}{|c|c|c|c|c|c|c|c|}
\hline \multicolumn{8}{|c|}{ bonds ] } \\
\hline & $a j$ & funct & r & $\mathrm{k}$ & & & \\
\hline 1 & 2 & 1 & $1.3497 e-01$ & $2.9865 e+05$ & ; & F1 & - $\mathrm{C} 1$ \\
\hline 2 & 3 & 1 & $1.3497 e-01$ & $2.9865 e+05$ & ; & $\mathrm{C} 1$ & $-F 2$ \\
\hline 2 & 4 & 1 & $1.3497 e-01$ & $2.9865 e+05$ & ; & $\mathrm{C} 1$ & $-F 3$ \\
\hline 2 & 5 & 1 & $1.8087 e-01$ & $1.9481 e+05$ & ; & $\mathrm{C} 1$ & $-\mathrm{s} 1$ \\
\hline 5 & 6 & 1 & $1.4660 e-01$ & $4.1254 \mathrm{e}+05$ & ; & s1 & -03 \\
\hline 5 & 7 & 1 & $1.4660 e-01$ & $4.1254 \mathrm{e}+05$ & ; & S1 & -04 \\
\hline 5 & 8 & 1 & $1.6723 e-01$ & $2.6535 e+05$ & ; & S1 & - N1 \\
\hline 8 & 9 & 1 & $1.5540 e-01$ & $3.6928 e+05$ & ; & N1 & $-\mathrm{s} 2$ \\
\hline 9 & 10 & 1 & $1.4533 e-01$ & $4.2903 e+05$ & ; & s2 & -01 \\
\hline 9 & 11 & 1 & $1.4533 e-01$ & $4.2903 e+05$ & ; & s2 & -02 \\
\hline 9 & 12 & 1 & $1.8075 e-01$ & $1.9539 e+05$ & ; & s2 & \\
\hline 12 & 13 & 1 & $1.3497 e-01$ & $2.9865 e+05$ & ; & $\mathrm{C} 2$ & - \\
\hline 12 & 14 & 1 & $1.3497 e-01$ & $2.9865 e+05$ & ; & C2 & $-F 6$ \\
\hline 12 & 15 & 1 & $1.3497 e-01$ & $2.9865 e+05$ & ; & $\mathrm{C} 2$ & \\
\hline
\end{tabular}

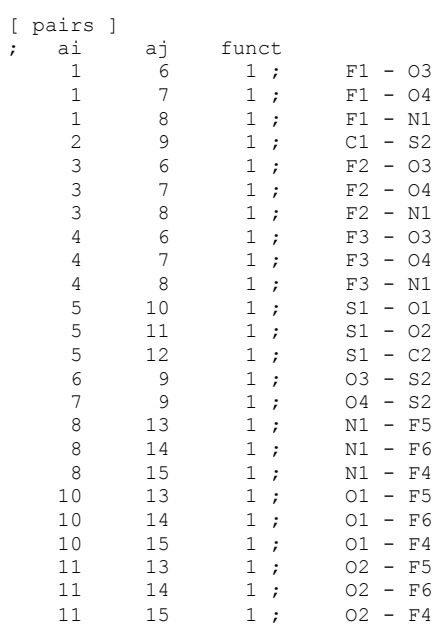

\begin{tabular}{|c|c|c|c|c|c|c|c|c|c|}
\hline$a i$ & & & & \\
\hline 1 & 2 & 3 & 1 & $1.0736 \mathrm{e}+02$ & $5.9321 \mathrm{e}+02$ & ; & F1 & $-\mathrm{C} 1$ & $-F 2$ \\
\hline 1 & 2 & 4 & 1 & $1.0736 \mathrm{e}+02$ & $5.9321 \mathrm{e}+02$ & ; & F1 & $-\mathrm{C} 1$ & $-F 3$ \\
\hline 1 & 2 & 5 & 1 & $1.0968 \mathrm{e}+02$ & $5.2819 e+02$ & ; & F1 & $-\mathrm{C} 1$ & - S1 \\
\hline 2 & 5 & 6 & 1 & $1.0785 \mathrm{e}+02$ & $5.4752 \mathrm{e}+02$ & ; & $\mathrm{C} 1$ & $-\mathrm{s} 1$ & -03 \\
\hline 2 & 5 & 7 & 1 & $1.0785 \mathrm{e}+02$ & $5.4752 \mathrm{e}+02$ & ; & $\mathrm{C} 1$ & $-\mathrm{S} 1$ & -04 \\
\hline 2 & 5 & 8 & 1 & $1.0219 e+02$ & $5.3614 \mathrm{e}+02$ & ; & $\mathrm{C} 1$ & $-\mathrm{s} 1$ & $-\mathrm{N} 1$ \\
\hline 3 & 2 & 4 & 1 & $1.0736 \mathrm{e}+02$ & $5.9321 e+02$ & ; & F2 & $-\mathrm{C} 1$ & $-F 3$ \\
\hline 3 & 2 & 5 & 1 & $1.0968 \mathrm{e}+02$ & $5.2819 e+02$ & ; & F2 & $-\mathrm{C} 1$ & $-\mathrm{S} 1$ \\
\hline 4 & 2 & 5 & 1 & $1.0968 e+02$ & $5.2819 e+02$ & ; & F3 & $-\mathrm{C} 1$ & $-\mathrm{s} 1$ \\
\hline 5 & 8 & 9 & 1 & $1.1918 \mathrm{e}+02$ & $5.7220 \mathrm{e}+02$ & ; & $\mathrm{S} 1$ & $-\mathrm{N} 1$ & $-\mathrm{s} 2$ \\
\hline 6 & 5 & 7 & 1 & $1.2141 \mathrm{e}+02$ & $6.0701 e+02$ & ; & 03 & $-\mathrm{S} 1$ & -04 \\
\hline 6 & 5 & 8 & 1 & $1.0965 \mathrm{e}+02$ & $5.8827 e+02$ & ; & 03 & $-\mathrm{s} 1$ & $-\mathrm{N} 1$ \\
\hline 7 & 5 & 8 & 1 & $1.0965 \mathrm{e}+02$ & $5.8827 e+02$ & ; & 04 & $-\mathrm{s} 1$ & $-\mathrm{N} 1$ \\
\hline 8 & 9 & 10 & 1 & $1.1910 e+02$ & $5.9287 e+02$ & ; & N1 & $-\mathrm{s} 2$ & -01 \\
\hline 8 & 9 & 11 & 1 & $1.1910 e+02$ & $5.9287 e+02$ & ; & N1 & $-\mathrm{s} 2$ & -02 \\
\hline 8 & 9 & 12 & 1 & $1.0819 \mathrm{e}+02$ & $1.3623 e+02$ & ; & N1 & $-\mathrm{s} 2$ & $-\mathrm{C} 2$ \\
\hline 9 & 12 & 13 & 1 & $1.0968 \mathrm{e}+02$ & $5.2819 e+02$ & ; & $\mathrm{s} 2$ & $-\mathrm{C} 2$ & - F5 \\
\hline 9 & 12 & 14 & 1 & $1.0968 \mathrm{e}+02$ & $5.2819 \mathrm{e}+02$ & ; & $\mathrm{s} 2$ & $-\mathrm{C} 2$ & $-F 6$ \\
\hline 9 & 12 & 15 & 1 & $1.0968 \mathrm{e}+02$ & $5.2819 \mathrm{e}+02$ & ; & $\mathrm{s} 2$ & $-\mathrm{C} 2$ & $-F_{4}$ \\
\hline 10 & 9 & 11 & 1 & $1.2005 e+02$ & $6.1580 e+02$ & ; & 01 & $-\mathrm{s} 2$ & -02 \\
\hline 10 & 9 & 12 & 1 & $1.0861 \mathrm{e}+02$ & $5.4702 e+02$ & ; & 01 & $-\mathrm{s} 2$ & - $\mathrm{C} 2$ \\
\hline 11 & 9 & 12 & 1 & $1.0861 \mathrm{e}+02$ & $5.4702 e+02$ & ; & 02 & $-\mathrm{s} 2$ & $-\mathrm{C} 2$ \\
\hline 13 & 12 & 14 & 1 & $1.0736 \mathrm{e}+02$ & $5.9321 \mathrm{e}+02$ & ; & F5 & $-\mathrm{C} 2$ & $-F 6$ \\
\hline 13 & 12 & 15 & 1 & $1.0736 \mathrm{e}+02$ & $5.9321 \mathrm{e}+02$ & ; & F5 & $-\mathrm{C} 2$ & $-F 4$ \\
\hline
\end{tabular}


14

12

15

[ dihedrals ] ; propers

$\begin{array}{cccr}\text { [ dihedrals } & \text { i propers } & \\ \mathbf{i} & j & \mathrm{k} & 1 \\ 1 & 2 & 5 & 6 \\ 1 & 2 & 5 & 7 \\ 1 & 2 & 5 & 8 \\ 2 & 5 & 8 & 9 \\ 3 & 2 & 5 & 6 \\ 3 & 2 & 5 & 7 \\ 3 & 2 & 5 & 8 \\ 4 & 2 & 5 & 6 \\ 4 & 2 & 5 & 7 \\ 4 & 2 & 5 & 8 \\ 5 & 8 & 9 & 10 \\ 5 & 8 & 9 & 11 \\ 5 & 8 & 9 & 12 \\ 6 & 5 & 8 & 9 \\ 7 & 5 & 8 & 9 \\ 8 & 9 & 12 & 13 \\ 8 & 9 & 12 & 14 \\ 8 & 9 & 12 & 15 \\ 10 & 9 & 12 & 13 \\ 10 & 9 & 12 & 14 \\ 10 & 9 & 12 & 15 \\ 11 & 9 & 12 & 13 \\ 11 & 9 & 12 & 14 \\ 11 & 9 & 12 & 15 \\ & & & \end{array}$

$1.0736 \mathrm{e}+02 \quad 5.9321 \mathrm{e}+02 ；$

func $\quad$ Co

0.60436
0.60436

0.60436
0.60436

30.54320

0.60436

0.60436

0.60436

0.60436

0.60436

55.78946

55.78946
55.78946

55.78946
30.54320

30.54320

30.54320
0.60436

0.60436

0.60436

0.60436

0.60436

0.60436

0.60436
0.60436

C1

1.81307

1. 81307

1.81307

22.17520

1.81307

1. 81307

1.81307

1.81307

1.81307

1.81307

1.81307

0.00000

0.00000
0.00000

22.17520

22.17520

1.81307

1.81307

1.81307

1.81307

1.81307

1.81307
1.81307

1.81307

1.81307
1.81307

1.81307
1.81307

$\mathrm{F} 6-\mathrm{C} 2$

- F4

C2 C3

$0.00000 \quad-2.41742$

0.00000

$0.00000 \quad 8.36800$

$.00000-2.41742$

$.00000-2.41742$

$0.00000-2.41742$

$0.00000-2.41742$

$.00000-2.41742$

$\begin{array}{ll}-55.78946 & 0.00000\end{array}$

0.00000

0.00000
0.00000

8.36800

$0.000000 \quad 8.36800$

$0.00000-2.41742$

$-2.41742$

$-2.41742$

0.00000

0.00000

$-2.41742$

-2.41742
-2.41742

$0.00000-2.41742$

0.00000

0.00000

-2.41742
-2.41742

-2.41742
-2.41742

$\mathrm{C} 4$

0.00000

0.00000

0.00000
0.00000

0.00000

0.00000

0.00000

0.00000

0.00000

0.00000

0.00000

0.00000

0.00000
0.00000

0.00000

0.00000

0.00000

0.00000

0.00000

0.00000

0.00000

0.00000

0.00000

0.00000

C5

$0.00000 ; \quad \mathrm{F} 1-\quad \mathrm{C} 1-\quad \mathrm{S} 1-\quad 03$

$\begin{array}{lllll}0.00000 ; & \mathrm{F} 1- & \mathrm{C} 1- & \mathrm{S} 1- & 04 \\ 0.00000 ; & \mathrm{F} 1- & \mathrm{C} 1- & \mathrm{S} 1- & \mathrm{N} 1\end{array}$

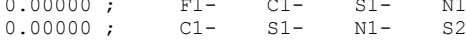

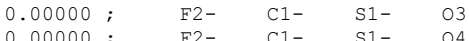

$0.00000 ; \quad \mathrm{F} 2-\mathrm{C} 1-\mathrm{S} 1-\mathrm{N} 1$

$0.00000 ; \quad \mathrm{F} 3-\mathrm{C} 1-\mathrm{S} 1-\mathrm{O}$

$0.00000 ; \quad \mathrm{F} 3-\mathrm{C} 1-\mathrm{S} 1-\mathrm{O}$

$0.00000 ; \quad \mathrm{F} 3-\mathrm{C} 1-\mathrm{S} 1-\mathrm{N} 1$

$0.0000 ; \quad \mathrm{S} 1-\quad \mathrm{N} 1-\mathrm{S} 2-\mathrm{O}$

$\begin{array}{lllll}0.00000 ; & \mathrm{S} 1- & \mathrm{N} 1- & \mathrm{S} 2- & \mathrm{O} 2 \\ 0.00000 ; & \mathrm{S} 1- & \mathrm{N} 1- & \mathrm{S} 2- & \mathrm{C} 2\end{array}$

$0.00000 ; \quad \mathrm{O} 3-\mathrm{S} 1-\mathrm{N} 1-\mathrm{S} 2$

$0.00000 ; \quad 04-\mathrm{S} 1-\quad \mathrm{N} 1-\mathrm{S} 2$

$\begin{array}{lllll}0.00000 ; & \mathrm{N} 1- & \mathrm{S} 2- & \mathrm{C} 2- & \mathrm{F} 5 \\ 0.00000 ; & \mathrm{N1} 1- & \mathrm{S} 2- & \mathrm{C} 2- & \mathrm{F} 6\end{array}$

$0.00000 ; \quad \mathrm{N} 1-\quad \mathrm{S} 2-\mathrm{C} 2-\mathrm{F} 4$

$0.00000 ; \quad 01-\quad \mathrm{S} 2-\quad \mathrm{C} 2-\quad \mathrm{F} 5$

$0.00000 ; \quad 01-\quad \mathrm{S} 2-\quad \mathrm{C} 2-\mathrm{F} 6$

$0.00000 ; \quad 01-\mathrm{S} 2-\mathrm{C} 2-\mathrm{F} 4$

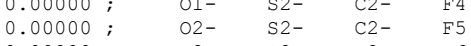

$\begin{array}{lllll}0.00000 ; & 02- & \mathrm{S} 2- & \mathrm{C} 2- & \mathrm{F} 6 \\ 0.00000 ; & \mathrm{O} 2- & \mathrm{S} 2- & \mathrm{C} 2- & \mathrm{F} 4\end{array}$

S5 